\title{
Структура и оптические свойства халькогенидного стеклообразного полупроводника As-Ge-Te
}

\author{
() А.И. Исаев, Х.И. Мамедова, С.И. Мехтиева, Р.И. Алекберов \\ Институт фризики Национальной академии наук Азербайджана, \\ Az-1143 Баку, Азербайджан \\ E-mail: physics.humay@mail.ru
}

Поступила в Редакцию 1 июня 2020 г.

В окончательной редакции 8 июня 2020 г.

Принята к публикации 8 июня 2020 г.

\begin{abstract}
Методами рентгеноструктурного анализа, рамановской спектроскопии, измерением оптического пропускания и плотности исследованы структура и оптические свойства пленки халькогенидного стеклообразного полупроводника $\mathrm{As}-\mathrm{Ge}-\mathrm{Te}$. Определены основные структурные элементы и химические связи, образующие аморфную матрицу, а также оптическая ширина запрещенной зоны. Полученные результаты объяснены с учетом основных принципов химического упорядочения и параметров ближнего порядка в расположении атомов.
\end{abstract}

Ключевые слова: координационные числа, когезионная энергия, плотности упаковки, компактности.

DOI: 10.21883/FTP.2020.10.49942.9454

\section{1. Введение}

В оптоэлектронике, волоконной оптике, нелинейных оптических системах и в устройствах памяти требуются материалы с высокой прозрачностью в инфракрасной (ИК) области спектра, с высоким значением показателя преломления и оптической нелинейности, а также химической стойкостью [1,2]. Халькогенидные стеклообразные полупроводники (ХСП), особенно, многокомпонентные, позволяют решить представленную задачу. Область прозрачности аморфных сульфидов и селенидов ограничена длинноволновым (многофононный) краем поглощения и простирается до 11-15 мкм [3]. Однако для некоторых целей требуются материалы с оптической прозрачностью за пределами указанных значений длины волн. Бинарные и тройные аморфные халькогениды, содержащие теллур (Ge-Te, As-Te, $\mathrm{Ge}-\mathrm{Ga}-\mathrm{Te}, \mathrm{Ge}-\mathrm{In}-\mathrm{Te}, \mathrm{As}-\mathrm{Ge}-\mathrm{Te}$ и т. д.), отличаются более широкими областями прозрачности [4,5]. Аморфный $\mathrm{As}-\mathrm{Ge}-\mathrm{Te}$ обладает широким окном прозрачности, охватывающим интервал длин волн 3-20 мкм [3]. Другим преимуществом указанного ХСП материала является высокое значение его показателя преломления $(n \sim 3.5$ при длине волны 1.55 мкм). Поэтому исследования структурных и оптических свойств указанного ХСП материала будут полезны при решении прикладных задач.

Считается признанным фактом, что макроскопические свойства некристаллических материалов контролируются особенностями ближнего порядка в расположении атомов. Химический состав ХСП материала, выбранного нами как объект исследования, позволяет, изменяя молярную долю составных элементов, добиться изменения параметров ближнего порядка (химические элементы, входящие в них, отличаются координационным числом, атомным радиусом, электроотрицательностью и т.д.).
Исследования физических свойств материалов, отличающихся параметрами ближнего порядка, позволят выяснить связь между параметрами ближнего порядка и макроскопическими свойствами [6,7], что необходимо для установления механизма физических процессов, происходящих в них.

Данная работа посвящена исследованию структуры, спектров комбинационного рассеяния света и коэффициента оптического поглощения ХСП системы As-Ge-Te.

\section{2. Методика эксперимента и изготовление образцов}

Синтез ХСП системы As-Ge-Te осуществлен в следующей последовательности: особо чистые элементарные вещества в требуемых атомных процентах наполнялись в кварцевые ампулы и после откачивания воздуха до давления $10^{-4}$ мм рт. ст., в течение 3 ч нагревались до температуры $900^{\circ} \mathrm{C}$ и выдерживались $\sim 12$ ч при этой температуре. С целью обеспечения однородности образцов синтез проводился во вращающейся печи, а охлаждение - в режиме выключенной печи. Пленки разной толщины, используемые в исследованиях, получены термическим испарением со скоростью 0.2-04 мкм/с на стеклянные подложки в вакууме при давлении $10^{-4}$ мм рт. ст. Плотность $(\rho)$ исследованных $\mathrm{XCП} \mathrm{систем} \mathrm{As-Ge-Se} \mathrm{измерялась} \mathrm{по} \mathrm{принципу} \mathrm{Ap-}$ химеда с использованием жидкости (воды) и вычислена по формуле

$$
\rho=\left[\frac{w_{0}}{\left(w_{0}-w_{L}\right)}\right] \rho_{L},
$$

где $w_{0}$ и $w_{L}-$ вес материала в воздухе и в жидкости (в воде). Плотность жидкости (воды) $\rho_{L}$ при комнатной температуре составляет 1 г/ $\mathrm{cm}^{3}$. Точность измерения не меньше \pm 0.02 г/ $\mathrm{cm}^{3}$. 


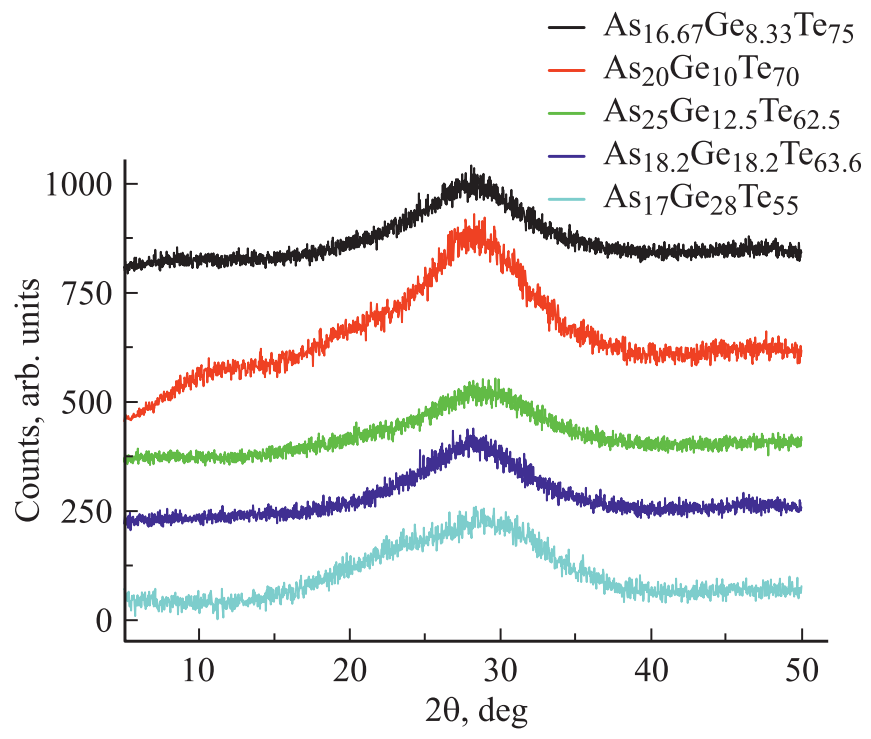

Рис. 1. Рентгеновские дифрактограммы ХСП системы $\mathrm{As}-\mathrm{Ge}-\mathrm{Te}$.

Исследования агрегатного состояния осажденных пленок проведены рентгеноструктурным анализом на порошковом дифрактометре D8 ADVANCE фирмы Брукер (Германия) в режиме $40 \mathrm{\kappa}, 40 \mathrm{MA}, 0<2 \theta<80^{\circ}$. На рис. 1 показаны кривые интенсивности дифракции рентгеновских лучей в пленках системы $\mathrm{As}-\mathrm{Ge}-\mathrm{Te}$ с разным содержанием составных элементов, которые хорошо согласуются с результатами работы [8]. Широкие максимумы, наблюдаемые в дифракционной картине, свидетельствуют об аморфности образцов.

Спектры комбинационного рассеяния света снимались на трехмерном конфокальном рамановском микроскопе Nanofinder 30 (Tokyo Instr.), длина волны возбуждения составляла 532 нм. Радиус сечения падающего на пленку лазерного луча $\sim 4$ мкм. Приемником излучения служила охлаждаемая $\mathrm{CCD}$-камера $\left(-70^{\circ} \mathrm{C}\right)$, работающая в режиме счета фотонов, время экспозиции составляло 1 мин.

Спектры пропускания тонкой пленки в диапазоне 190-1100 нм были получены с использованием ультрафиолетового видимого инфракрасного спектрофотометpa (SPEKOL-1500). Спектрофотометр устанавливали с шириной щели 2 нм. Все данные были получены при комнатной температуре.

\section{3. Экспериментальные результаты и их обсуждение}

На рис. 2 представлен спектр комбинационного рассеяния света системы As-Ge-Te. Как видно, изменением химического состава спектр претерпевает существенное изменение; либо изменяется интенсивность различных максимумов, либо некоторые максимумы исчезают и вместо них появляются другие.
В спектре $\mathrm{As}_{16.67} \mathrm{Ge}_{8.33} \mathrm{Te}_{75}$ наблюдаются две полосы: в интервале $100-135 \mathrm{~cm}^{-1}$ с максимумами при 121 и $137 \mathrm{~cm}^{-1}$ и более широкая полоса, охватывающая интервал частоты $135-245 \mathrm{~cm}^{-1}$ с максимумом при $153 \mathrm{~cm}^{-1}$. Следует отметить, что максимум при $123 \mathrm{~cm}^{-1}$ был наблюден также в работах [8-10] и приписан к колебаниям тетраэдров $\mathrm{GeTe}_{4}$ (симметричный режим растяжения $\mathrm{A}_{1}$ ), а также симметричным изгибам колебаний пирамид $\mathrm{AsTe}_{3}$ (мода $\mathrm{A}_{1}$ ). Пик при $120 \mathrm{~cm}^{-1}$ также наблюден в комбинационном спектре тригонального теллура [10]. Основываясь на этих фактах, авторы работ $[10,11]$ пришли к выводу, что полоса, охватывающая пик при $123 \mathrm{~cm}^{-1}$, может иметь смешанный характер с некоторым вкладом колебаний Те-Те. Полоса с максимумами при 136-141 см${ }^{-1}$, наблюдаемая во всех химических составах, причем более интенсивно в спектре образцов 1 и 2 (с большим содержанием теллура), также наблюдена авторами работ $[8,12]$ и отнесена к колебаниям коротких неупорядоченных цепей Те, которая в аморфном теллуре экспериментально наблюдалась при $157 \mathrm{~cm}^{-1}[13,14]$, где частота колебаний смещается из-за дальнодействующего взаимодействия между цепями. Полоса с максимумами при $159 \mathrm{~cm}^{-1}$ была приписана антисимметричным изгибным колебаниям пирамид $\mathrm{AsTe}_{3}$ (Е-мода) [10], а также $\mathrm{A}_{1}$-моде $\mathrm{GeTe}_{4}$ с четырехгранным разделением краев или же Те-Те, как в аморфном теллуре (неупорядоченные цепи) [14]. Слабая полоса в интервале частот $185-195 \mathrm{~cm}^{-1}$ также наблюдена авторами работ $[8,13]$ и связывалась с колебаниями октаэдрических единиц $\mathrm{GeTe}_{3}$. Наблюдаемая очень слабая полоса в интервале $220-230 \mathrm{~cm}^{-1}$ связывалась модой антисимметричных валентных колебаний $\mathrm{GeTe}_{4}-n \mathrm{Ge}_{n}$, однако нельзя исключить колебания структурных элементов с участием атомов мышьяка. Учитывая это, авторы работ $[8,13,15]$ считают, что полосы при 185-195 и $220-230 \mathrm{~cm}^{-1}$ частично относятся к симметричным (режи $\mathrm{A}_{1}$ ) и антисимметричным (режим Е) валентным колебаниям пирамид $\mathrm{AsTe}_{3}$. Как видно из спектра, с

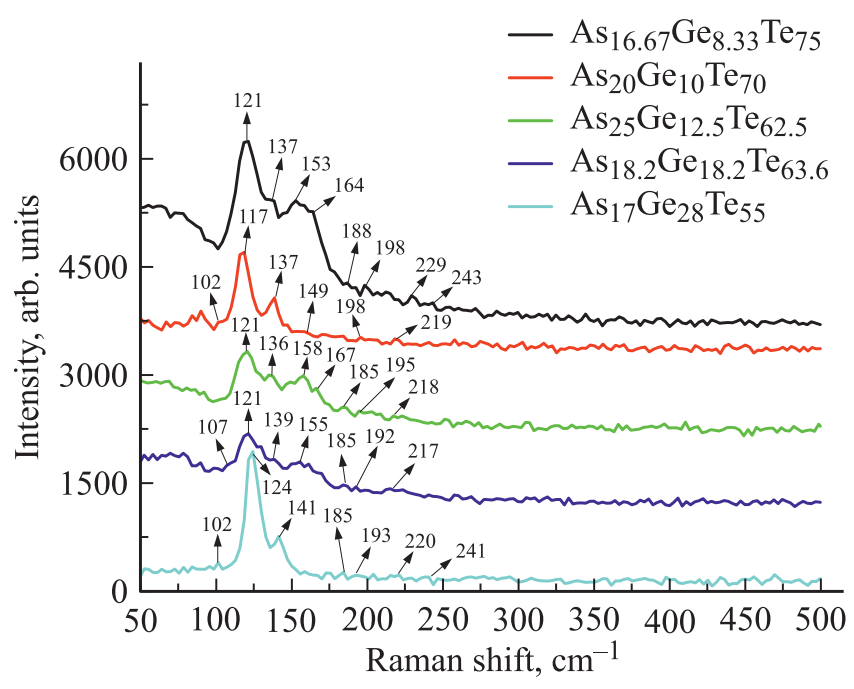

Pис. 2. Спектры комбинационного рассеяния света системы As-Ge-Te. 
Таблица 1. Оценки среднего значения координационного числа $Z$, плотности упаковки $(\varkappa)$, компактности $(\delta)$, среднего значения атомного объема $\left(V_{a}\right)$ и экспериментально определяемые значения плотности $(\rho)$ исследованных образцов

\begin{tabular}{|c|c|c|c|c|c|c|c|c|}
\hline $\begin{array}{c}\text { № } \\
\text { образца }\end{array}$ & $\begin{array}{c}\text { Номинальный состав. } \\
\text { Предполагаемая химическая } \\
\text { формула }\end{array}$ & $Z$ & $R$ & $\begin{array}{c}\rho, \\
\Gamma / \mathrm{cm}^{3}\end{array}$ & $\begin{array}{c}x, \\
\left(\cdot 10^{22} \mathrm{~cm}^{-3}\right)\end{array}$ & $\delta$ & $\begin{array}{c}V_{a}, \\
\left(\mathrm{~cm}^{3} / \text { Моль }\right)\end{array}$ & $\begin{array}{c}E_{g}, \text { эB } \\
\text { (эксперимент) }\end{array}$ \\
\hline 1 & $\begin{array}{l}\mathrm{As}_{16.67} \mathrm{Ge}_{8.33} \mathrm{Te}_{75} \\
{\left[0.417\left(\mathrm{As}_{0.4} \mathrm{Te}_{0.6}\right)\right] \cdot} \\
{\left[0.252\left(\mathrm{Ge}_{0.333} \mathrm{Te}_{0.666}\right)\right]\left[\mathrm{Te}_{0.332}\right]}\end{array}$ & 2.33 & 1.800 & 6.22 & 3.28 & 0.016 & 18.36 & 0.97 \\
\hline 2 & $\begin{array}{l}\mathrm{As}_{20} \mathrm{Ge}_{10} \mathrm{Te}_{70} \\
{\left[0.5\left(\mathrm{As}_{0.4} \mathrm{Te}_{0.6}\right)\right] \cdot} \\
{\left[0.303\left(\mathrm{Ge}_{0.333} \mathrm{Te}_{0.666}\right)\right]\left[\mathrm{Te}_{0.198}\right]}\end{array}$ & 2.40 & 1.400 & 6.01 & 3.24 & -0.015 & 18.58 & 0.99 \\
\hline 3 & $\begin{array}{l}\mathrm{As}_{25} \mathrm{Ge}_{12.5} \mathrm{Te}_{62.5} \\
{\left[0.625\left(\mathrm{As}_{0.4} \mathrm{Te}_{0.6}\right)\right] \cdot} \\
{\left[0.379\left(\mathrm{Ge}_{0.333} \mathrm{Te}_{0.666}\right)\right]}\end{array}$ & 2.50 & 1.000 & 5.14 & 2.88 & -0.155 & 20.90 & 1.03 \\
\hline 4 & $\begin{array}{c}\mathrm{As}_{18.2} \mathrm{Ge}_{18.2} \mathrm{Te}_{63.6} \\
{\left[0.452\left(\mathrm{As}_{0.4} \mathrm{Te}_{0.6}\right)\right] \cdot} \\
{\left[0.547\left(\mathrm{Ge}_{0.333} \mathrm{Te}_{0.666}\right)\right]\left[\mathrm{As}_{0.019}\right]}\end{array}$ & 2.55 & 9.998 & 6.03 & 3.36 & -0.003 & 17.92 & 1.02 \\
\hline 5 & $\begin{array}{c}\mathrm{As}_{17} \mathrm{Ge}_{28} \mathrm{Te}_{55} \\
{\left[0.826\left(\mathrm{Ge}_{0.333} \mathrm{Te}_{0.666}\right)\right]} \\
{\left[0.012\left(\mathrm{Ge}_{0.429} \mathrm{As}_{0.571}\right)\right]\left[\mathrm{As}_{0.163}\right]}\end{array}$ & 2.73 & 0.675 & 5.96 & 3.47 & -0.002 & 17.35 & 1.05 \\
\hline
\end{tabular}

ростом содержания мышьяка и германия максимумы в высокочастотной области постепенно ослабляются или исчезают. Действительно, в образцах с порядковыми номерами 2, 3 и 4 полоса при $141-230 \mathrm{~cm}^{-1}$ сильно ослабляется и полностью исчезает в последнем образце 5. Такое изменение в спектре прежде всего можно связывать с уменьшением доли структурных элементов с участием атомов теллура. В спектре состава $\mathrm{As}_{17} \mathrm{Ge}_{28} \mathrm{Te}_{55}$ проявляется максимум при $124 \mathrm{~cm}^{-1}$. Последнее можно объяснить тем, что в составах с большими содержаниями теллура широкий максимум образовался из-за колебаний нескольких элементов с участием атомов теллура, а также неупорядоченных цепей теллура. В составе $\mathrm{As}_{17} \mathrm{Ge}_{28} \mathrm{Te}_{55}$ из-за малого содержания атомов теллура вклад колебаний неупорядоченных цепей не учитывается, вследствие чего ширина полосы уменьшается. Узкий максимум, полученный при $141 \mathrm{~cm}^{-1}$, также был наблюден в комбинационном спектре $[16,17]$ и приписывался к Е-моде кристаллического Те. Слабые максимумы в интервале $\sim 102-107 \mathrm{~cm}^{-1}$, наблюдаемые в образцах 2-5, также наблюдены авторами [16] в стеклообразном $\mathrm{As}-\mathrm{Ge}-\mathrm{Se}$ и приписывались суммарным вкладам колебаний связи As-As и $\mathrm{Ge}-\mathrm{As}$. Обычно для прогноза о виде существующих химических связей, установления связи между ними и наблюдаемыми особенностями электронных процессов используют основные принципы метода химической связи (Chemical bond approaches (CBA) model) [8]. Согласно CВА, вероятность образования гетерополярных связей превосходит вероятность образования гомеополярных связей, причем вначале формируются самые прочные связи (связи с высокими энергиями). Последовательность образования указанных связей соответствует по- следовательности убывания энергии до тех пор, пока не будет удовлетворена доступная валентность атомов.

В таблице представлен предполагаемый номинальный состав исследованных материалов. Обычно состояние многокомпонентных материалов характеризуется средним координационным числом $Z$ и параметром $R$, определяемым отношением числа возможных ковалентных связей атомов халькогена к числу возможных ковалентных связей нехалькогенидных атомов [18]. Значения указанных параметров вычисляются по формулам

$$
\begin{gathered}
Z=4 X_{\mathrm{Ge}}+3 X_{\mathrm{As}}+2 X_{\mathrm{Te}}, \\
R=\frac{2 X_{\mathrm{Te}}}{4 X_{\mathrm{Ge}}+3 X_{\mathrm{As}}},
\end{gathered}
$$

где $X_{\mathrm{Ge}}, X_{\mathrm{As}}$ и $X_{\mathrm{Te}}$ - молярные доли элементов в ХСП составе. Полученные значения представлены в табл. 1.

Используя экспериментальные данные по плотности, вычислены значения таких параметров локальной структуры исследованных материалов, как плотность упаковки $(\varkappa)$, компактность $(\delta)$ и среднее значение атомного объема $\left(V_{a}\right)$ по формулам $(2),(3)$ и (5).

$$
\begin{gathered}
\varkappa=\frac{\rho N_{A}}{\Sigma_{i} x_{i} A_{i}}, \\
V_{a}=\frac{1}{\rho} \Sigma_{i} x_{i} A_{i}, \\
\delta=\frac{\Sigma_{i}^{\left(x_{i} A_{i}\right)} / \rho_{i}-\Sigma_{i}^{\left(x_{i} A_{i}\right)} / \rho}{\Sigma_{i}^{\left(x_{i} A_{i}\right)} / \rho},
\end{gathered}
$$

где $x_{i}, A_{i}$ и $\rho_{i}$ - молярная доля, атомная масса и плотность элементов, входящих в ХСП состав соответственно, $N_{\mathrm{A}}$ - число Авагадро. Полученные значения указанных параметров представлены в табл. 1. 
Значение $R=1$ соответствует стехиометрическому составу, состоящему только из энергетически выгодных гетерополярных связей. Для химических составов, богатых халькогеном (теллуром) (образцы 1 и 2 в табл. 1), в которых наряду с существованием гетерополярных связей $\mathrm{As}-\mathrm{Te}$ и $\mathrm{Ge}-\mathrm{Te}$ присутствуют и гомеополярные связи Те-Те, значения $R$ больше единицы. В химических составах, отличающихся нехваткой атомов халькогена, для полного удовлетворения валентных требований нехалькогенных атомов (образцы 4 и 5 в табл. 1) значения $R$ будут меньше единицы. Эти составы должны отличаться высокой концентрацией гомеополярных связей As-As (из-за высокого значения энергии связи $\mathrm{Ge}-\mathrm{Te}$ валентные требования большинства атомов германия удовлетворяются).

Особенности комбинационного спектра исследованных материалов удовлетворительно согласуются с принципами СВА. Действительно, согласно СВА, аморфная матрица образца 3 должна образоваться в основном из полностью сшитых тетраэдрических $\left(\mathrm{Ge}\left[\mathrm{Te}_{1 / 2}\right]_{4}\right)$ и пирамидальных $\left(\mathrm{AsTe}_{3 / 2}\right)$ структурных единиц, которые содержат энергетически выгодные гетерополярные связи. Однако анализ комбинационного спектра показал также наличие коротких неупорядоченных цепей Те (при $157 \mathrm{~cm}^{-1}$ ), пирамид $\mathrm{AsTe}_{3}$, октаэдрических единиц $\mathrm{GeTe}_{3}$ и т.д. Такое поведение свидетельствует о некотором отклонении от принципы CВА, т.е. существует частичная химическая неупорядоченность. Кроме того, наблюдение максимума при $141 \mathrm{~cm}^{-1}$ в спектре образца 5 может быть связано с гомеополярной связью Те-Те между пирамидальными и октаэдрическими структурными элементами.

С целью определения оптической ширины запрещенной зоны исследованы спектры пропускания ХСП системы As $-\mathrm{Ge}-\mathrm{Te}$ в области высоких значений коэффициента поглощения. Значения $\alpha$ вычислены по формуле $\alpha=\frac{1}{d} \ln \frac{(1-R)^{2}}{\tau}$ и полученные результаты в виде зависимости от $(\alpha h v)^{1 / 2}$ представлены на рис. 3. где $R$ и $T-$ коэффициенты отражения и пропускания исследованных слоев, а $d-$ их толщина. Линейный ход указанной зависимости в достаточно широком интервале энергии фотона свидетельствует о том, что в исследованных материалах электронные переходы соответствуют непрямым разрешенным переходам. Экстраполируя линейную часть кривых на нулевое поглощение, определена оптическая ширина запрещенной зоны (табл. 2).

Численные значения ширины запрещенной зоны вычислены согласно формуле, предложенной авторами работы $[19]$ для многокомпонентных стекол $\left(A_{x} B_{y} C_{z}\right)$ :

$$
E_{g}^{r}=x E_{g}(A)+y E_{g}(B)+z E_{g}(C),
$$

где $E_{g}(A), E_{g}(B)$ и $E_{g}(C)$ - ширины запрещенной зоны элементарных веществ, входящих в многокомпонентный материал: $E_{g}(\mathrm{Te})=2.05$ эB,$E_{g}(\mathrm{As})=1.2$ э $\mathrm{B}$, $E_{g}(\mathrm{Ge})=1$ эB $[20]$.

Важными параметрами, характеризующими аморфную матрицу неупорядоченных материалов, являются среднее значение энергии связи и когезионная энергия.

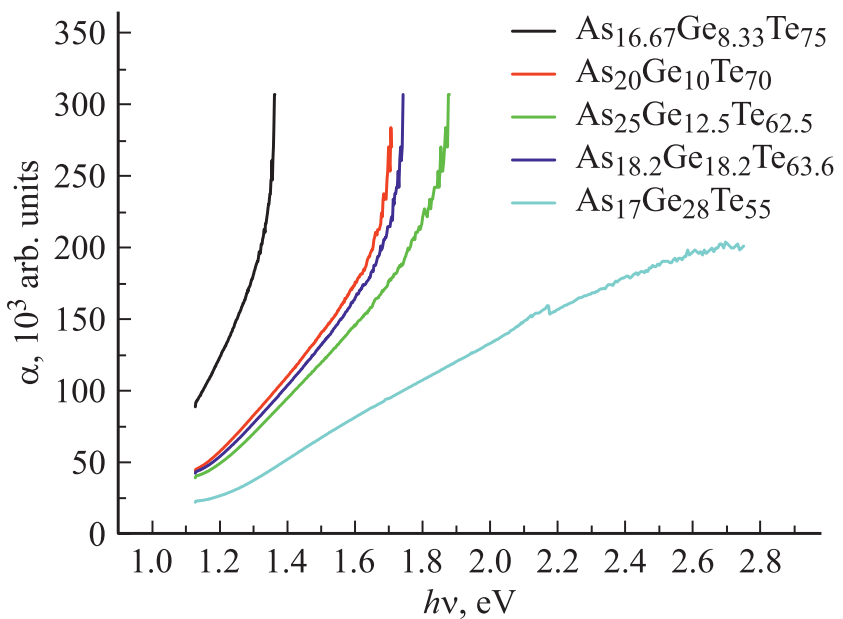

Pис. 3. Спектры оптического поглощения ХСП системы $\mathrm{As}-\mathrm{Ge}-\mathrm{Te}$.

Средние значения энергии связи исследованных материалов вычислены по формулам, предложенным авторами работы [21] для сложных халькогенидных материалов:

$$
E_{r}=E_{c}+E_{r m},
$$

где $E_{c}-$ средняя энергия связи поперечной сшивки на атом и $E_{r m}-$ средняя энергия связи на атом оставшейся матрицы.

Значения $E_{c}$ вычисляются по формуле:

$$
\begin{array}{ll}
E_{c}=P_{r} E_{h b} & R>1, \\
E_{c}=P_{p} E_{h b} & R<1,
\end{array}
$$

где $P_{r}$ и $P_{p}$ - степень сшивки на атом, $E_{h b}-$ среднее значение энергии гетерополярной связи. Для стекла с композицией $\mathrm{As}_{x} \mathrm{Ge}_{y} \mathrm{Te}_{z} E_{h b}$ - вычисляется по формуле

$$
E_{h b}=x \cdot Z_{\mathrm{As}} \cdot E_{\mathrm{As}-\mathrm{Te}} y \cdot Z_{\mathrm{Ge}} \cdot E_{\mathrm{Ge}-\mathrm{Te}} /\left(x \cdot Z_{\mathrm{As}}+y \cdot Z_{\mathrm{Ge}}\right),
$$

где $E_{\mathrm{As}-\mathrm{Te}}$ и $E_{\mathrm{Ge}-\mathrm{Te}}-$ энергия гетерополярных связей. $P_{r}$ и $P_{p}$ вычисляются из формулы:

$$
\begin{gathered}
P_{r}=\left(x \cdot Z_{\mathrm{As}}+y \cdot Z_{\mathrm{Ge}}\right) /(x+y+z) \quad \text { при } \quad R>1 \\
P_{p}=z \cdot Z_{\mathrm{Te}} /(x+y+z) \quad R<1 . \quad(12)
\end{gathered}
$$

Энергия $E_{m}$ получается из формулы

$$
\begin{gathered}
E_{r m}=2\left(\frac{1}{2} Z-P_{r}\right)^{E_{C-C}} / Z \quad R>1, \\
E_{r m}=2\left(\frac{1}{2} Z-P_{p}\right)^{E_{<>}} / Z \quad R>1,
\end{gathered}
$$

где $E_{C-C}-$ энергия связи между халькогенными атомами, $E_{<>}-$среднее значение энергии связи между нехалькогенными атомами (As и Ge) в ХCП составах, где $R<1$. 
Таблица 2. Химические связи, их процентные доли и рассчитанные значения по формуле (6), а также энергии ожидаемых химический связей

\begin{tabular}{l|c|c|c|c|c|c|c|c}
\hline \multicolumn{1}{c|}{ Состав } & $\mathrm{Ge}-\mathrm{Te}$ & $\mathrm{As}-\mathrm{Te}$ & $\mathrm{Te}-\mathrm{Te}$ & $\mathrm{Ge}-\mathrm{As}$ & $\mathrm{As}-\mathrm{As}$ & $C E$ & $E_{m}$ & $\begin{array}{c}E_{g}, \text { эВ } \\
\text { (рассчитанная) }\end{array}$ \\
\hline $\mathrm{As}_{16.67} \mathrm{Ge}_{8.33} \mathrm{Te}_{75}$ & 0.288 & 0.430 & 0.285 & & & 33.04 & 37.04 & 0.96 \\
$\mathrm{As}_{20} \mathrm{Ge}_{10} \mathrm{Te}_{70}$ & 0.336 & 0.500 & 0.165 & & & 36.15 & 39.15 & 0.95 \\
$\mathrm{As}_{25} \mathrm{Ge}_{12.5} \mathrm{Te}_{62.5}$ & 0.403 & 0.600 & & & & 34.03 & 34.13 & 0.99 \\
$\mathrm{As}_{18.2} \mathrm{Ge}_{18.2} \mathrm{Te}_{63.6}$ & 0.571 & 0.425 & & & 0.001 & 34.72 & 44.04 & 0.97 \\
$\mathrm{As}_{17} \mathrm{Ge}_{28} \mathrm{Te}_{55}$ & 0.806 & & & 0.015 & 0.18 & 36.20 & 46.62 & 0.98 \\
Энергия химических \\
связей, ккал/моль
\end{tabular}

Учитывая ожидаемые химические связи и их процентную долю в исследованных материалах, представленных в табл. 2, оценена когезионная энергия по формуле (15) [22,23], и полученные результаты представлены в табл. 2:

$$
C E=\Sigma_{i} c_{i} E_{i}
$$

где $c_{i}$ и $E_{i}$ - количество и энергия ожидаемых связей.

Согласно [24], происхождение валентной зоны в халькогенидных стеклах связано с состояниями электронов неподеленной пары (LP electron states ), а зоны проводимости - антисвязывающими ( $\sigma^{*}$ - antibonding states) состояниями $p$-орбиталей атомов халькогена. Экспериментально определяемые значения оптической ширины запрещенной зоны соответствуют энергетическим расстояниям между верхними границами LP-состояний и дна $\sigma^{*}$-состояний. Ясно, что значения $E_{g}$ будут изменяться в результате изменения указанных состояний. Изменения в значении ширины запрещенной зоны происходят в результате изменения энергии связи, когезионной энергии, плотности вещества и плотности упаковки. Увеличение значения координационного числа $(Z)$ соответствует усилению взаимодействия между атомами [25], что в свою очередь приводит к усилению разделения между состояниями $\sigma$ и $\sigma^{*}$, и таким образом увеличивается значение $E_{g}$. Кроме того, как видно из табл. 2, с ростом $Z$ уменьшается плотность вещества, вследствие чего увеличивается край зоны проводимости в результате увеличивается $E_{g}$. Уменьшение плотности вещества является признаком ослабления эффектов ближнего порядка, что также обусловливает рост значения $E_{g}$. Как видно из табл. 2 , по мере увеличения $Z$ относительной доли химических связей с высокими энергиями увеличиваются значение когезионной энергии и среднее значение энергии связи, а также экспериментально определяемые значения ширины запрещенной зоны. Исключение происходит только для стехиометрического состава $\left(\mathrm{As}_{25} \mathrm{Ge}_{12.5} \mathrm{Te}_{62.5}\right)$, где значения $R$ и $Z$ составляют 1 и 2.5 соответственно, т. е. указанный состав для данной системы соответствует химическому порогу. Как уже было отмечено, аморфная матрица образца, соответствующего химическому порогу, образуется в основном из полностью сшитых тетраэдрических $\left(\mathrm{Ge}\left[\mathrm{Te}_{1 / 2}\right]_{4}\right)$ и
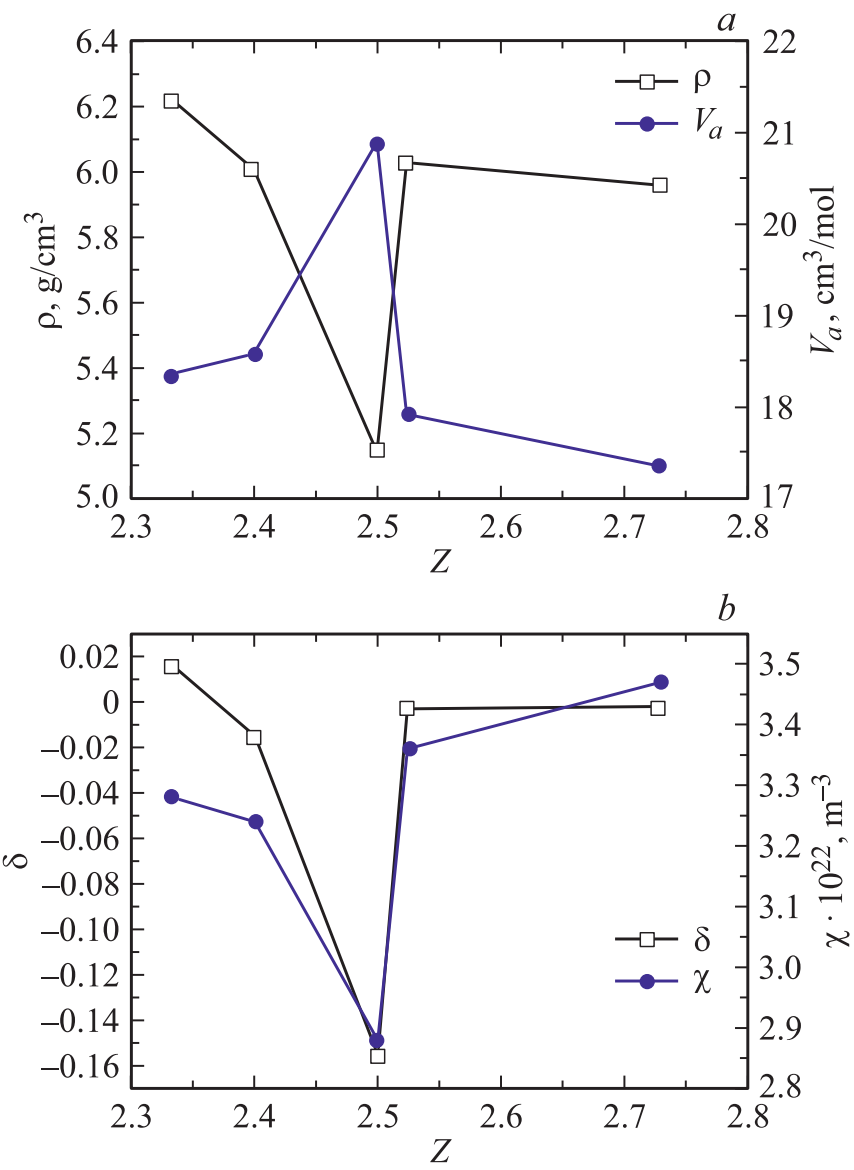

Рис. 4. Зависимость плотности вещества $(\rho)$ и среднего значения атомного объема $\left(V_{a}\right)(a)$, плотности упаковки $(\chi)$ и компакности $(\delta)(b)$ от среднего значения координационного числа для системы $\mathrm{As}-\mathrm{Ge}-\mathrm{Te}$.

пирамидальных $\left(\mathrm{AsTe}_{3 / 2}\right)$ структурных единиц, т.е. из 3D структурных единиц. Считается, что при значениях координационного числа $Z=2.5$ образуется полностью сшитая трехмерная матрица и происходит структурный переход 2D $\rightarrow 3 \mathrm{D}$. Согласно [26], чтобы достичь полностью сшитой трехмерной матрицы, требуется самый большой средний атомный объем. При этом плотность вещества, плотность упаковки и компакность должны 
иметь минимальное значение, что действительно было наблюдено (рис. 4).

\section{4. Заключение}

Синтезированы ХСП составы $\mathrm{As}_{16.67} \mathrm{Ge}_{8.33} \mathrm{Te}_{75}$, $\mathrm{As}_{20} \mathrm{Ge}_{10} \mathrm{Te}_{70}, \quad \mathrm{As}_{25} \mathrm{Ge}_{12.5} \mathrm{Te}_{62.5}, \quad \mathrm{As}_{18.2} \mathrm{Ge}_{18.2} \mathrm{Te}_{63.6}$ и $\mathrm{As}_{17} \mathrm{Ge}_{28} \mathrm{Te}_{55}$, и термическим напылением в вакууме получены их слои толщиной 1-3 мки. Аморфность слоев подтверждена рентгеноструктурным анализом.

Анализом спектра комбинационного рассеяния света установлены основные структурные элементы и химические связи, образующие аморфную матрицу системы $\mathrm{As}-\mathrm{Ge}-\mathrm{Te}$. Аморфная матрица стехиометрического состава $\mathrm{As}_{25} \mathrm{Ge}_{12.5} \mathrm{Te}_{62.5}(R=1)$ в основном состоит из полностью сшитых тетраэдрических $\left(\mathrm{Ge}\left[\mathrm{Te}_{1 / 2}\right]_{4}\right)$ и пирамидальных $\left(\mathrm{AsTe}_{3 / 2}\right)$ структурных единиц. Однако в спектре также наблюдался пик, соответствующий колебаниям пирамид $\mathrm{AsTe}_{3}$, октаэдрических единиц $\mathrm{GeTe}_{3}$ и связи Те-Те, что указывает на некоторые отклонения от правила СВА. В спектрах образцов с избытком атомов теллура (образцы 1 и 2) появляются пики в высокочастотной области, а с нехваткой теллура (образцы 4 и 5) - в низкочастотной. Первые соответствуют структурным элементам с участием атомов теллура и неупорядоченной цепи из теллура, а вторые связьям As-As и As-Ge.

Изменения оптической ширины запрешенной зоны в зависимости от координационного числа связывались с изменениями энергии связи, когезионной энергии, плотности вещества и коэффициента упаковки атомов.

Достижение максимального значения среднего атомного объема, минимального значения плотности вещества, коэффициента упаковки и компактности атомов для состава $\mathrm{As}_{25} \mathrm{Ge}_{12.5} \mathrm{Te}_{62.5}(R=1, Z=2.5)$ связывалось структурным переходом $2 \mathrm{D} \rightarrow 3 \mathrm{D}$.

\section{Конфликт интересов}

Авторы заявляют, что у них нет конфликта интересов.

\section{Список литературы}

[1] A. Zakery, S. Elliott. J. Non-Cryst. Sol., 330 (1-3), 1 (2003).

[2] J.S. Sanghera, I.D. Aggarwal. J. Non-Cryst. Sol., 256-257, 6 (1999).

[3] B.J. Eggleton, B. Luther-Davies, K. Richardson. Nature Photonics, 5, 141 (2011).

[4] B. Bureau. Solid State Sci., 10, 427 (2008).

[5] Z.Y. Yang, P. Lucas. J. Am. Ceram. Soc., 92, 2920 (2009).

[6] А.И. Исаев, С.И. Мехтиева, Х.И. Мамедова, Р.И. Алекберов. ФТП, 11, 1532 (2019).

[7] А.И. Исаев, С.И. Мехтиева, Х.И. Мамедова1, Р.И. Алекберов. Физика и химия стекла, 46 (1), 64 (2020).

[8] P. Hawlová, F. Verger, V. Nazabal, R. Boidin, P. Němec. Scientific Rep. (Nature Publishing Group), 5, 9310 ( 2015).

[9] K. Andrikopoulos, S.N. Yannopoulos, G. Voyiatzis. J. Phys.: Condens. Matter, 18, 965(2006).
[10] S. Sen, E.L. Gjersing, B.G. Aitken. J. Non-Cryst. Sol., 356, 2083 (2010).

[11] A.S. Pine, G. Dresselhaus. Phys. Rev. B, 4, 356 (1971).

[12] H. Krebs, P. Fischer. Discussions of the Faraday Society, 50, 35 (1970).

[13] M.H. Brodsky, J.E. Smith, Y.Yacoby, R.J. Gambino. Physica Status Solidi B, 52, 609 (1972).

[14] I. Voleska, J. Akola, P. Jovari, J. Gutwirth, T. Wagner, T. Vasileiadis, S.N. Yannopoulos, R.O. Jones. Phys. Rev. B, 86, 094108 (2012).

[15] R.T. Ananth Kumar, Hussein A. Mousa, P. Chithra Lekha, Saleh T. Mahmoud, N. Qamhieh. J. Physics: Conf. Ser., 869, 012018 (2017).

[16] C. Zha, R.P. Wang, A. Smith, A. Prasad, R.A. Jarvis, B. LutterDavies. J. Mater Sci.: Mater. Electron., 18, S389 (2007).

[17] A.S. Hassanien, A.A. Akl. J. Non-Cryst. Sol., 428, 1129 (2015).

[18] L. Tichy, H. Ticha. Mater. Lett., 21, 313 (1994).

[19] K. Shimakawa. J. Non-Cryst. Sol., 43, 229 (1981).

[20] Н. Мотт, Э. Девис. Электронные процессы в некристаллических веществах (М., Мир, 1982).

[21] L. Tichy, H. Ticha. J. Non-Cryst. Sol., 189, 141 (1995).

[22] V. Pamukchieva, A. Szekeres, K. Todorova, M. Fabian, E. Svab, Zs. Revay, L. Szentmiklosi. J. Non-Cryst. Sol., 325, 2485 (2009).

[23] L. Pauling. The Nature of the Chemical Bond (Cornell University Press, Ithaca, N. Y., 1960).

[24] M. Kastner. Phys. Rev. Lett., 28, 355 (1972).

[25] J.P. Deneufville, H. Rockstad. In: J. Stuke, W. Brenig (eds). Amorphous and Liquid Semiconductors (Taylor and Francis, London, 1974) p. 419.

[26] P.S. Giridhar, L. Narasimham, S. Mahadevan. J. Non-Cryst. Sol., 43, 29 (1981).

Редактор Г.А. Оганесян

\section{Structure and optical properties of the As-Ge-Te chalcogenide glassy semiconductor}

\section{A.I. Isayev, H.I. Mammadova, S.I. Mekhtiyeva, R.I. Alekberov}

Institute of Physics, Azerbaijan National Academy of Sciences, Az-1143 Baku, Azerbaijan

Abstract The structure and optical properties of the $\mathrm{As}-\mathrm{Ge}-\mathrm{Te}$ chalcogenide glass semiconductor (CGS) were studied by $X$-ray diffraction, analysis Raman spectroscopy, the optical transmission and density measurements. Have been determined the main structural elements and chemical bonds forming an amorphous matrix, as well as the optical band gap. The results are explained taking into account the basic principles of chemical ordering and short-range order parameters in the arrangement of atoms. 